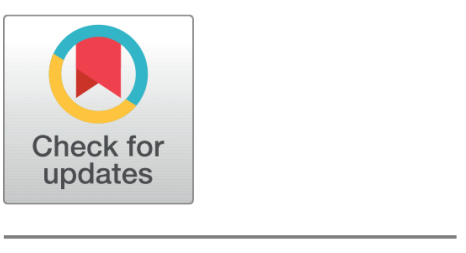

OPEn ACCESS

Received: 16.10.2020

Accepted: 01.01.2021

Published: 13.01.2021

Citation: Savita, Sahrawat RK, Malik M (2021) Stresses in a monoclinic elastic plate placed upon an irregular monoclinic elastic half space. Indian Journal of Science and Technology 14(1): 55-70. https://doi. org/10.17485/IJST/v14i1.1874

* Corresponding author.

Tel: 8397883690

savitadhankhar67@gmail.com

Funding: HRDG Council of Scientific \& Industrial Research for

sanctioning SRF scholarship with File No. (09/1063/0012/2017)

Competing Interests: None

Copyright: (c) 2021 Savita et al. This is an open access article distributed under the terms of the Creative Commons Attribution License, which permits unrestricted use, distribution, and reproduction in any medium, provided the original author and source are credited.

Published By Indian Society for Education and Environment (iSee)

ISSN

Print: 0974-6846

Electronic: 0974-5645

\section{Stresses in a monoclinic elastic plate placed upon an irregular monoclinic elastic half space}

\author{
Savita ${ }^{1}{ }^{*}$, Ravinder Kumar Sahrawat ${ }^{1}$, Meenal Malik ${ }^{2}$ \\ 1 Department of Mathematics, Deenbandhu Chhotu Ram University of Science and \\ Technology, Murthal, 131039, India. Tel.: 8397883690 \\ 2 Department of Mathematics, All India Jat Heroes Memorial College, Rohtak, 124001, India
}

\section{Abstract}

Objective: To study the stresses due to strip load in a monoclinic elastic plate placed over an irregular monoclinic elastic half space. Method: Antiplane strain problem with different interfacing boundary conditions namely "perfectly welded interfacing, smooth-rigid interfacing, rough-rigid interfacing" has been considered and Fourier Transformation is used on the equilibrium equations to obtain the solutions. Findings: The displacements and stresses are obtained for each boundary condition and the variation of shearing stresses with horizontal distance are studied due to different size of rectangular shaped irregularity at any point of the medium consisting of monoclinic elastic plate placed upon an irregular monoclinic elastic half space. The comparison between the stresses for different types of interfacing has been made graphically. Novelty: The static deformation of two or more connected elastic mediums with irregularities due to surface loads has been studied earlier but just a few have showed graphic representation of stresses with irregularity in different sizes. Keeping in mind the shortcomings of earlier work done, the present paper visualizes shearing stresses in both theoretical and graphical manners completely.

Keywords: Monoclinic; strip loading; anti plane strain; rectangular irregularity

\section{Introduction}

The concept of dislocation theory has been proved a helpful tool in the study of earth crust deformation due to surface loads or any internal sources. Several researchers dealt mathematically with the elastic residual fields based on the dislocation theory describing static deformation of the earth crust associated with an earthquake. Crampin ${ }^{(1)}$ investigated for the seismic anisotropy in the earth crust, which was a beginning of anisotropic crystal study but the seismic anisotropy was an unfamiliar concept for many geophysicists. Keeping this in mind, he tried to suggest a consistent terminology having simple and descriptive meanings and made the concept of anisotropy easier to remember and understood. It was found that the two or three dimensional static deformation of an elastic material due to surface loads was important for understanding 
the behaviour of the elastic medium at continental margins and mountain roots in the earth structure. Following this criteria, the problem of static deformation present in the elastic mediums had been studied by many researchers, for e.g Pan ${ }^{(2)}$ solved the static deformation problem of a transversely isotropic layered half space with different type of dislocation sources which contained the solutions to two and three dimensional source problem for the corresponding isotropic media. The formulation developed in this paper could be evaluated numerically to study the effect of anisotropy as well as of earth layering on the static fields. Garg and Singh ${ }^{(3)}$ obtained the stresses as a result of shear line load acting at the boundary of a semi infinite elastic model consisting of an isotropic elastic layer lying over an isotropic elastic half space and concluded that quasi-static stresses differ significantly from the corresponding static stresses in pure elastic medium. Madan and Garg ${ }^{(4)}$ obtained the displacements and stresses at any point of an orthotropic horizontal elastic layer interfacing in three different ways with a base due to a very long inclined strike slip fault. Garg et.al. ${ }^{(5)}$ examined the static deformation in an orthotropic elastic medium due to a dip slip fault of infinite length and finite width. As mentioned by Crampin ${ }^{(1)}$ there was monoclinic symmetry in the earth crust. Monoclinic symmetry of systems of cracks may be found near the surface of the earth where lithostatic pressure has not closed cracks. Monoclinic crystal system is one of the seven (triclinic, monoclinic, orthorhombic, tetragonal, trigonal, hexagonal and cubic) crystal systems. The monoclinic crystal system has been described by three vectors of unequal length in which two are perpendicular to each other. In mathematical description, an elastic plate can be related to the earth crust and the coupling between the elastic layer and the elastic half space can be perfectly bonded, smooth-rigid and rough-rigid. Singh et.al. ${ }^{(6)}$ formulated the problem of two-dimensional static deformation of a monoclinic elastic medium using the Eigen value approach and Fourier transform method. Madan ${ }^{(7)}$ obtained solution to the problem of static deformation in a monoclinic elastic medium with considerations of welded half space in the form of a layer with a base under different kind of interfacing in the antiplane strain state of equilibrium. For convenience, many researchers formulated an elastic problem under the consideration of perfect isotropic elastic and homogeneity for the elastic medium instead of irregular half space. But under these assumptions certain ambiguities arose between theory and observations that led to point out the need for reformulating some problems under less restrictive assumptions. Madan ${ }^{(8)}$ considered a problem in which the static deformation of transversely isotropic elastic half space with rectangular shaped irregularity present on the interfacing of the elastic mediums caused by surface loads. Following the above approaches and results, Madan ${ }^{(9)}$ obtained the 2-Dimensional static deformation of an irregular orthotropic elastic medium with the assumption of rectangular shape and parabolic shape irregularities due to normal line loads. Madan ${ }^{(10)}$ discussed the effect of irregularity and also effect of anisotropy on the shear stresses due to strip loads on an

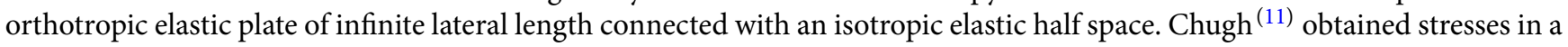
transversely isotropic elastic plate due to strip-loading as a contour map in anti-plane strain state under perfectly bonding boundary condition. Verma et al. ${ }^{(12)}$ obtained the plane strain problem of the deformation of a homogeneous, isotropic, poroelastic layer of uniform thickness overlying a homogeneous, isotropic, elastic half-space due to two-dimensional seismic sources buried in the elastic half-space. The effect of the compressibility of the fluid and solid constituents of the poroelastic layer is to decrease and increase the magnitude of the pore pressure respectively has been concluded. Tiersten ${ }^{(13)}$ developed many experimental and theoretical results in both two-dimensional and three-dimensional visualization for the measure of arbitrary elasticity tensors and also for permeability in mountains, rocks and fluids.

In this paper, we have obtained closed form analytical expressions for the displacements and stresses in a horizontal infinite monoclinic elastic layer placed over an infinite irregular monoclinic elastic half space. The variation of shearing stresses has been studied graphically with different sizes of irregularity.

\section{Basic equation}

The equilibrium equations in the cartesian co-ordinate system for zero body forces are

$$
\begin{aligned}
& \tau_{x x, x}+\tau_{x y, y}+\tau_{x z, z}=0 \\
& \tau_{y x, x}+\tau_{y y, y}+\tau_{y z, z}=0 \\
& \tau_{z x, x}+\tau_{z y, y}+\tau_{z z, z}=0
\end{aligned}
$$

where $\tau_{x x}, \tau_{y y}, \tau_{z z}$ are normal stresses and $\tau_{x y}, \tau_{y z}, \tau_{z x}$ are shearing stresses. 
The stress-strain relations for a monoclinic elastic medium in co-ordinate plane are given by Crampin ${ }^{(1)}$

$$
\left.\begin{array}{c}
\tau_{x x}=c_{11} e_{x x}+c_{12} e_{y y}+c_{13} e_{z z}+c_{14} e_{y z} \\
\tau_{y y}=c_{12} e_{x x}+c_{22} e_{y y}+c_{23} e_{z z}+c_{24} e_{y z} \\
\tau_{z z}=c_{13} e_{x x}+c_{23} e_{y y}+c_{33} e_{z z}+c_{34} e_{y z} \\
\tau_{y z}=c_{14} e_{x x}+c_{24} e_{y y}+c_{34} e_{z z}+c_{44} e_{y z} \\
\tau_{z x}=c_{55} e_{z x}+c_{56} e_{x y} \\
\tau_{x y}=c_{56} e_{z x}+c_{66} e_{x y}
\end{array}\right\}
$$

Also, we have the following strain displacement relations

$$
e_{x x}=\frac{\partial u}{\partial x}, e_{y y}=\frac{\partial v}{\partial y}, e_{z z}=\frac{\partial w}{\partial z}, e_{x y}=\frac{1}{2}\left(\frac{\partial u}{\partial y}+\frac{\partial v}{\partial x}\right), e_{y z}=\frac{1}{2}\left(\frac{\partial v}{\partial z}+\frac{\partial w}{\partial y}\right), e_{z x}=\frac{1}{2}\left(\frac{\partial w}{\partial x}+\frac{\partial u}{\partial z}\right)
$$

where $e_{x x}, e_{y y}, e_{z z}$ are normal strain components and $e_{x y}, e_{y z}, e_{z x}$ are shearing strain components. $(u, v, w)$ are the displacement components in cartesian coordinate plane. The two suffix quantities $c_{i j}(i, j=1,2,3,4,5,6)$ are the elastic constants.

The displacements and stresses for a monoclinic elastic material in anti-plane strain equilibrium state in the $y z-$ plane is

$$
\left.\begin{array}{c}
u=u(y, z), \quad v=0, \quad w=0 \\
e_{x x}=e_{y y}=e_{z z}=e_{y z}=0 \\
e_{x y}=\frac{1}{2} \frac{\partial u}{\partial y}, \quad e_{x z}=\frac{1}{2} \frac{\partial u}{\partial z} \\
\tau_{x y}=c_{56} \frac{\partial u}{\partial z}+c_{66} \frac{\partial u}{\partial y} \\
\tau_{z x}=c_{55} \frac{\partial u}{\partial z}+c_{56} \frac{\partial u}{\partial y}
\end{array}\right\}
$$

Using equations 4-6, the equilibrium equations (2)-(3) are identically zero and equation (1) reduced to

$$
\frac{\partial^{2} u}{\partial y^{2}}+2 \frac{c_{56}}{c_{66}} \frac{\partial^{2} u}{\partial y \partial z}+\frac{c_{55}}{c_{66}} \frac{\partial^{2} u}{\partial z^{2}}=0
$$

We defined the Fourier Transform of $f(x, y)$ as

$$
\bar{f}(x, s)=\int_{-\infty}^{\infty} f(x, y) e^{i y s} d y
$$

So, the Inverse Fourier Transform is defined as

$$
f(x, y)=\frac{1}{2 \pi} \int_{-\infty}^{\infty} \bar{f}(x, s) e^{-i y s} d s
$$

\section{Formulation of the problem}

We consider the cartesian co-ordinate system $(x, y, z)$. Here, $z$-axis has been taken horizontally and $y$-axis along vertically downward. An infinite monoclinic elastic plate of thickness ' $Y$ ' lying horizontally over an irregular infinite monoclinic elastic half-space. The irregularity is assumed to be rectangular in shape. The origin of Cartesian co-ordinate system $(x, y, z)$ is taken at the upper boundary of the plate. The plate occupying the region $(0 \leq y \leq Y)$ is described as medium I whereas the region $y>Y$ is described as medium II. (as shown in Figure 1). 


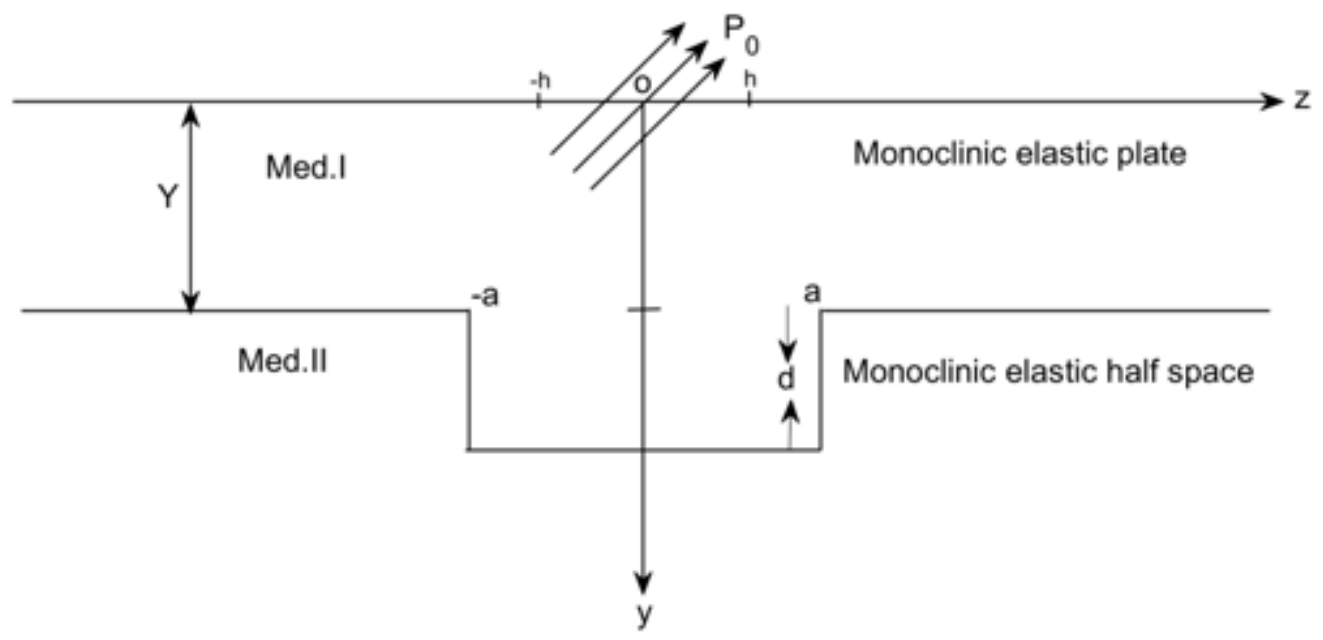

Fig 1.

Suppose a shear load $P_{0}$ per unit area is acting over the strip $(z \mid \leq h$ of the surface $y=0$ in the positive $x$-direction.

So, the boundary condition (at the surface $y=0$ ) is,

$$
\tau_{\mathrm{xy}}=\left\{\begin{array}{c}
-\mathrm{P}_{0}:|\mathrm{z}|<h \\
0:|z|>h
\end{array}\right.
$$

The equation of irregularity is represented as:

$$
y=\varepsilon f(z)=\left\{\begin{array}{l}
d:|z|<a \\
0:|z|>a
\end{array}\right.
$$

where $\varepsilon=\frac{d}{2 a} \ll 1$ is the perturbation factor.

We are considering the interface between the layer and the half-space as "perfectly welded, smooth rigid, and rough rigid". The boundary conditions for different types of coupling are

For perfectly welded contact:

$$
\begin{aligned}
& u\left(y=Y^{-}\right)=u\left(y=Y^{+}\right) \\
& \tau_{x y}\left(y=Y^{-}\right)-i \varepsilon f^{\prime}(z) \tau_{z x}\left(y=Y^{-}\right)=\tau_{x y}\left(y=Y^{+}\right)-i \varepsilon f^{\prime}(z) \tau_{z x}\left(y=Y^{+}\right)
\end{aligned}
$$

For smooth-rigid contact:

$$
\tau_{x y}=0 \text { at } y=Y
$$

For rough-rigid contact:

$$
u=0 \text { at } y=Y
$$

Now, taking the Fourier Transform of equation (7), we have

$$
\frac{d^{2} \bar{u}}{d y^{2}}-2\left(i s \frac{c_{56}}{c_{66}}\right) \frac{d \bar{u}}{d y}-\frac{c_{55}}{c_{66}} s^{2} \bar{u}=0
$$

The solution of above ordinary differential equation is

$$
\bar{u}=\left(A e^{m_{1}|s| y}+B e^{-m_{1}|s| y}\right) e^{-i\left(m_{2} y\right) s}
$$

where $m_{1}=\sqrt{m_{3}-m_{2}^{2}}, m_{2}=\frac{c_{56}}{c_{66}}, m_{3}=\frac{c_{55}}{c_{66}}$ 
Now, taking the Inverse Fourier Transform of equation (13), the displacements and stresses for (Med. I) are

$$
u^{\mathrm{I}}=\frac{1}{2 \pi} \int_{-\infty}^{\infty}\left(A e^{m_{1}|s| y}+B e^{-m_{1}|s| y}\right) e^{-i\left(z-m_{2} y\right) s} d s
$$

Using equation (14) in equation (6), we have

$$
\begin{gathered}
\tau_{x y}^{I}=\frac{T_{1}}{2 \pi} \int_{-\infty}^{\infty}\left(A e^{m_{1}|s| y}-B e^{-m_{1}|s| y}\right) e^{-i\left(z-m_{2} y\right)}|s| d s \\
\tau_{x z}^{\mathrm{I}}=\frac{T_{1}}{2 \pi}\left(m_{2} \int_{-\infty}^{\infty}\left(A e^{m_{1}|s| y}-B e^{-m_{1}|s| y}\right) e^{-i\left(z-m_{2} y\right) s}|s| d s\right. \\
\left.-i m_{1} \int_{-\infty}^{\infty}\left(A e^{m_{1}|s| y}-B e^{-m_{1}|s| y}\right) e^{-i\left(z-m_{2} y\right) s} s\right) d s
\end{gathered}
$$

where $T_{1}=m_{1} c_{66}$

Using the Fourier Transform technique on the boundary condition (8), we have

$$
\tau_{x y}^{I}=\frac{-P_{0}}{\pi} \int_{-\infty}^{\infty} \frac{\sin (s h)}{s} e^{-i s z} d s
$$

Now, from equations (15) and (17), we have

$$
A-B=\frac{-2 P_{0}}{T_{1}}\left(\frac{\sin (s h)}{s|s|}\right)
$$

The displacement and stresses for med. II (i.e for $y>Y$ ) are

$$
u^{\mathrm{II}}=\frac{1}{2 \pi} \int_{-\infty}^{\infty} C\left(e^{-m_{1}^{\prime}|s| y} e^{-l\left(z-m_{2}^{\prime} y\right) s}\right) d s
$$

the coefficient of $e^{m_{1}|s| y}$ is taken as zero, otherwise, $u \rightarrow \infty$ as $y \rightarrow \infty$

$$
\begin{gathered}
\tau_{x y}^{\mathrm{II}}=\frac{-T_{1}^{\prime}}{2 \pi} \int_{-\infty}^{\infty} C\left(e^{-m_{1}^{\prime}|s| y} e^{-i\left(z-m_{2}^{\prime} y\right) s}\right)|s| d s \\
\tau_{x z}^{\mathrm{II}}=\frac{-T_{1}^{\prime}}{2 \pi} \int_{-\infty}^{\infty} C\left(m_{2}^{\prime}|s|+i m_{1}^{\prime} s\right)\left(e^{-m_{1}^{\prime}|s| y} e^{-i\left(z-m_{2}^{\prime} y\right) s}\right) d s
\end{gathered}
$$

where $T_{1}^{\prime}=m_{1}^{\prime} c_{66}^{\prime}, \quad m_{1}^{\prime}=\sqrt{m_{3}^{\prime}-m_{2}^{\prime 2}}, \quad m_{2}^{\prime}=\frac{c_{56}^{\prime}}{c_{66}^{\prime}}, m_{3}^{\prime}=\frac{c_{55}^{\prime}}{c_{66}^{\prime}}$ and A, B, C may be functions of ' $s$ ' and determined from the boundary conditions.

By applying the Fourier transform technique on equation (9), we have

$$
f(z)=\operatorname{sign}(a-z)+\operatorname{sign}(a+z)
$$

where 'sign' is the Signum function.

\section{Perfect contact}

When the interfacing between the plate and half-space at $y=\varepsilon f(z)$ is welded contact, then condition (12), (14), (15), (16), (19), (20), and (21) yield

$$
\left(A e^{m_{1}|s| \varepsilon f(z)}+B e^{-m_{1}|s| \varepsilon f(z)}\right) e^{i m_{2} \varepsilon f(z) s}-c e^{-m_{1}^{\prime}|s| \varepsilon f(z)} e^{i m_{2}^{\prime} \varepsilon f(z) s}=0
$$




$$
\begin{gathered}
A e^{m_{1}|s| \varepsilon f} e^{i m_{2} \varepsilon f s}\left(T_{1} S_{1}-i \varepsilon f^{\prime} m_{2} T_{1} S_{1}-\varepsilon f^{\prime} m_{1}\right) \\
-B e^{-m_{1}|s| \varepsilon f} e^{i m_{2} \varepsilon f s}\left(T_{1} S_{1}-i \varepsilon f^{\prime} m_{2} T_{1} S_{1}+\varepsilon f^{\prime} m_{1}\right) \\
+C e^{-m_{1}^{\prime}|s| \varepsilon f} e^{i m_{2}^{\prime} \varepsilon f s}\left(T_{1}^{\prime} S_{1}-i \varepsilon f^{\prime} m_{2}^{\prime} T_{1}^{\prime} S_{1}+\varepsilon f^{\prime} m_{1}^{\prime}\right)=0
\end{gathered}
$$

Solving equations (18), (26) and (27), we have the values

$$
\begin{gathered}
A=\frac{2 P_{0}}{T_{1}} \frac{\sin (s h)}{s|s|}\left(\frac{\left(S_{1} V-\varepsilon f^{\prime} V_{3}+i \varepsilon f^{\prime} S_{1} V_{2}\right) e^{-2 m_{1}|s| \varepsilon f}}{\left(S_{1}-i \varepsilon f^{\prime} S_{1} V_{1}+\varepsilon f^{\prime} V_{3}\right)-\left(S_{1} V-\varepsilon f^{\prime} V_{3}+i \varepsilon f^{\prime} S_{1} V_{2}\right) e^{-2 m_{1}|s| \varepsilon f}}\right) \\
B=\frac{2 P_{0}}{T_{1}} \frac{\sin (s h)}{s|s|}\left(1-\frac{\left(S_{1} V-\varepsilon f^{\prime} V_{3}+i \varepsilon f^{\prime} S_{1} V_{2}\right) e^{-2 m_{1}|s| \varepsilon f}}{\left(S_{1}-i \varepsilon f^{\prime} S_{1} V_{1}+\varepsilon f^{\prime} V_{3}\right)-\left(S_{1} V-\varepsilon f^{\prime} V_{3}+i \varepsilon f^{\prime} S_{1} V_{2}\right) e^{-2 m_{1}|s| \varepsilon f}}\right) \\
C=\frac{4 P_{0}}{T_{1}} \frac{\sin (s h)}{s|s|}\left(\frac{S_{1}\left(1+i \varepsilon f^{\prime} m_{2}\right) e^{-(\alpha|s|+i \beta s) \varepsilon f}}{\left(S_{1}-i \varepsilon f^{\prime} S_{1} V_{1}+\varepsilon f^{\prime} V_{3}\right)-\left(S_{1} V-\varepsilon f^{\prime} V_{3}+i \varepsilon f^{\prime} S_{1} V_{2}\right) e^{-2 m_{1}|s| \varepsilon f}}\right)
\end{gathered}
$$

where $V=\frac{T-1}{T+1}, V_{1}=\frac{T_{1} m_{2}+T_{1}^{\prime} m_{2}^{\prime}}{T_{1}+T_{1}^{\prime}}, \quad V_{2}=\frac{T_{1} m_{2}-T_{1}^{\prime} m_{2}^{\prime}}{T_{1}+T_{1}^{\prime}}, \quad V_{3}=\frac{m_{1}^{\prime}-m_{1}}{T_{1}+T_{1}^{\prime}}$

$$
T=\frac{T_{1}}{T_{1}^{\prime}}, \quad \alpha=m_{1}-m_{1}^{\prime}, \quad \beta=m_{2}-m_{2}^{\prime}
$$

Substituting the values for A, B, C from (28)-(30) in equations (14)-(16) for med. I and in equations (19)-(21) for med. II and also substituting the value for $\mathrm{f}(\mathrm{z})$ from (22), we will obtain the following results for displacements and stresses.

For med. I(i.e. , for $\leq y \leq Y)$

$$
\begin{aligned}
& u^{\mathrm{I}}=\frac{P_{0}}{\pi T_{1}} \int_{-\infty}^{\infty} \frac{\sin (s h)}{s|s|}\left(e^{-m_{1}|s| y}+\sum_{n=1}^{\infty} V^{n} e^{-m_{1}|s|(2 n \varepsilon f-y)}-\sum_{n=1}^{\infty} V^{n} e^{-m_{1}|s|(2 n \varepsilon f+y)}\right) e^{-i\left(z-m_{2} y\right) s} d s \\
& \tau_{x y}^{\mathrm{I}}=-\frac{P_{0}}{\pi}\left[\tan ^{-1} \frac{2 h m_{1} y}{\left(z-m_{2} y\right)^{2}+m_{1}^{2} y^{2}-h^{2}}-\sum_{n=1}^{\infty} V^{n}\left(\tan ^{-1} \frac{2 h m_{1}(2 n \varepsilon f+y)}{\left(z-m_{2} y\right)^{2}+m_{1}^{2}(2 n \varepsilon f+y)^{2}-h^{2}}\right.\right. \\
& \left.\left.+\tan ^{-1} \frac{2 h m_{1}(2 n \varepsilon f-y)}{\left(z-m_{2} y\right)^{2}+m_{1}^{2}(2 n \varepsilon f-y)^{2}-h^{2}}\right)\right] \\
& \tau_{x z}^{I}=-\frac{P_{0} m_{2}}{\pi}\left[\tan ^{-1} \frac{2 h m_{1} y}{\left(z-m_{2} y\right)^{2}+m_{1}^{2} y^{2}-h^{2}}-\sum_{n=1}^{\infty} V^{n}\left(\tan ^{-1} \frac{2 h m_{1}(2 n \varepsilon f+y)}{\left(z-m_{2} y\right)^{2}+m_{1}^{2}(2 n \varepsilon f+y)^{2}-h^{2}}\right.\right. \\
& \left.\left.+\tan ^{-1} \frac{2 h m_{1}(2 n \varepsilon f-y)}{\left.\left(z-m_{2} y\right)^{2}+m_{1}^{2}(2 n \varepsilon f)-y\right)^{2}-h^{2}}\right)\right]+\frac{m_{1} P_{0}}{2 \pi}\left[\log \frac{\left[\left(z-m_{2} y\right)+h\right]^{2}+\left(m_{1} y\right)^{2}}{\left[\left(z-m_{2} y\right)-h\right]^{2}+\left(m_{1} y\right)^{2}}\right. \\
& \left.-\sum_{n=1}^{\infty} V^{n}\left(\log \frac{\left[\left(z-m_{2} y\right)+h\right]^{2}+\left[m_{1}(2 n \varepsilon f+y)\right]^{2}}{\left[\left(z-m_{2} y\right)-h\right]^{2}+\left[m_{1}(2 n \varepsilon f+y)\right]^{2}}+\log \frac{\left[\left(z-m_{2} y\right)+h\right]^{2}+\left[m_{1}(2 n \varepsilon f-y)\right]^{2}}{\left[\left(z-m_{2} y\right)-h\right]^{2}+\left[m_{1}(2 n \varepsilon f-y)\right]^{2}}\right)\right]
\end{aligned}
$$

For med. II (i.e. $y>Y$ )

$$
\begin{aligned}
u^{\mathrm{II}}= & \frac{2 P_{0}}{\pi T_{1}}\left(\frac{T}{T+1}\right) \int_{-\infty}^{\infty} \frac{\sin (s h)}{s|s|}\left(e^{-m_{1}|s| y-(\alpha|s|+i \beta s) \varepsilon f}+\right. \\
& \left.\sum_{n=1}^{\infty} V^{n} e^{\left.-m_{1}|s|(2 n \varepsilon f+y)-(\alpha|s|+\imath \beta s) \varepsilon f\right)}\right) e^{-i\left(z-m_{2}^{\prime} z y\right) s} d s
\end{aligned}
$$




$$
\begin{aligned}
\tau_{x y}^{\mathrm{II}}= & -\frac{2 P_{0}}{\pi(1+T)}\left[\tan ^{-1} \frac{2 h\left(m_{1}^{\prime} y+\alpha \varepsilon f\right)}{\left(z-m_{2}^{\prime} y+\imath \beta \varepsilon f\right)^{2}+\left(m_{1}^{\prime} y+\alpha \varepsilon f\right)^{2}-h^{2}}\right. \\
& \left.+\sum_{n=1}^{\infty} V^{n} \tan ^{-1} \frac{2 h\left(\left(2 n m_{1}+\alpha\right) \varepsilon f+m_{1}^{\prime} y\right)}{\left(z-m_{2}^{\prime} y+\imath \beta \varepsilon f\right)^{2}+\left(\left(2 n m_{1}+\alpha\right) \varepsilon f+m_{1}^{\prime} y\right)^{2}-h^{2}}\right] \\
\tau_{x z}^{\mathrm{II}}= & -\frac{2 P_{0} m_{2}^{\prime}}{(1+T) \pi}\left(\tan ^{-1} \frac{2 h\left(m_{1}^{\prime} y+\alpha \varepsilon f\right)}{\left(z-m_{2}^{\prime} y+\imath \beta \varepsilon f\right)^{2}+\left(m_{1}^{\prime} y+\alpha \varepsilon f\right)^{2}-h^{2}}\right. \\
& \left.+\sum_{n=1}^{\infty} V^{n} \tan ^{-1} \frac{2 h\left(\left(2 n m_{1}+\alpha\right) \varepsilon f+m_{1}^{\prime} y\right)}{\left(z-m_{2}^{\prime} y+\imath \beta \varepsilon f^{2}+\left(\left(2 n m_{1}+\alpha\right) \varepsilon f+m_{1}^{\prime} y\right)^{2}-h^{2}\right.}\right) \\
& +\frac{P_{0} m_{1}^{\prime}}{\pi(1+T)}\left(\log \frac{\left(z-m_{2}^{\prime} y+\imath \beta \varepsilon f+h\right)^{2}+\left(m_{1}^{\prime} y+\alpha \varepsilon f\right)^{2}}{\left(z-m_{2}^{\prime} y+\imath \beta \varepsilon f-h\right)^{2}+\left(m_{1}^{\prime} y+\alpha \varepsilon f\right)^{2}}+\right. \\
& \left.+\sum_{n=1}^{\infty} V^{n} \log \frac{\left(z-m_{2}^{\prime} y+\imath \beta \varepsilon f+h\right)^{2}+\left(m_{1}^{\prime} y+\left(2 n m_{1}+\alpha\right) \varepsilon f\right)^{2}}{\left(z-m_{2}^{\prime} y+\imath \beta \varepsilon f-h\right)^{2}+\left(m_{1}^{\prime} y+\left(2 n m_{1}+\alpha\right) \varepsilon f\right)^{2}}\right)
\end{aligned}
$$

\section{Smooth rigid-interface}

When the interfacing between plate and half-space at $\mathrm{y}=\mathrm{Y}$ is smooth-rigid, without loss of generality, on taking $T \longrightarrow \infty$ i.e, $V=1$ in equations (31)-(33) for med. I and in equations (34)-(36) for med. II. we will obtain the following results for displacements and stresses.

For med. I(i.e., for $\leq y \leq Y$ )

$$
\begin{aligned}
u^{\mathrm{I}}= & \frac{P_{\mathrm{o}}}{\pi T_{1}} \int_{-\infty}^{\infty} \frac{\sin (s h)}{s|s|}\left(e^{-m_{1}|s| y}+\sum_{n=1}^{\infty} e^{-m_{1}|s|(2 n \varepsilon f-y)}-\sum_{n=1}^{\infty} e^{-m_{1}|s|(2 n \varepsilon f+y)}\right) e^{-i\left(z-m_{2} y\right) s} d s \\
\tau_{x y}^{I}= & -\frac{P_{0}}{\pi}\left[\tan ^{-1} \frac{2 h m_{1} y}{\left(z-m_{2} y\right)^{2}+m_{1}^{2} y^{2}-h^{2}}-\sum_{n=1}^{\infty}\left(\tan ^{-1} \frac{2 h m_{1}(2 n \varepsilon f+y)}{\left(z-m_{2} y\right)^{2}+m_{1}^{2}(2 n \varepsilon f+y)^{2}-h^{2}}\right.\right. \\
& \left.\left.+\tan ^{-1} \frac{2 h m_{1}(2 n \varepsilon f-y)}{\left(z-m_{2} y\right)^{2}+m_{1}^{2}(2 n \varepsilon f-y)^{2}-h^{2}}\right)\right] \\
\tau_{x z}^{\mathrm{I}}=- & \frac{P_{0} m_{2}}{\pi}\left[\tan ^{-1} \frac{2 h m_{1} y}{\left(z-m_{2} y\right)^{2}+m_{1}^{2} y^{2}-h^{2}}-\sum_{n=1}^{\infty}\left(\tan ^{-1} \frac{2 h m_{1}(2 n \varepsilon f+y)}{\left(z-m_{2} y\right)^{2}+m_{1}^{2}(2 n \varepsilon f+y)^{2}-h^{2}}\right.\right. \\
+ & \left.\left.\tan ^{-1} \frac{2 h m_{1}(2 n \varepsilon f-y)}{\left(z-m_{2} y\right)^{2}+m_{1}^{2}(2 n \varepsilon f-y)^{2}-h^{2}}\right)\right]+\frac{m_{1} P_{0}}{2 \pi}\left[\log \frac{\left[\left(z-m_{2} y\right)+h\right]^{2}-m_{1} y^{2}}{\left[\left(z-m_{2} y\right)-h\right]^{2}+m_{1} y^{2}}\right. \\
& -\sum_{n=1}^{\infty}\left(\log \frac{\left[\left(z-m_{2} y\right)+h\right]^{2}+\left[m_{1}(2 n \varepsilon f+y)\right]^{2}}{\left[\left(z-m_{2} y\right)-h\right]^{2}+\left[m_{1}(2 n \varepsilon f+y)\right]^{2}}+\log \frac{\left[\left(z-m_{2} y\right)+h\right]^{2}+\left[m_{1}(2 n \varepsilon f-y)\right]^{2}}{\left[\left(z-m_{2} y\right)-h\right]^{2}+\left[m_{1}(2 n \varepsilon f-y)\right]^{2}}\right]
\end{aligned}
$$

For med. II (i.e. $y>Y$ )

$$
\begin{gathered}
u^{\mathrm{II}}=\frac{2 P_{0}}{\pi T_{1}} \int_{-\infty}^{\infty} \frac{\sin (s h)}{s|s|}\left(e^{-m_{1}|s| y}+\sum_{n=1}^{\infty} e^{-m_{1}|s| y-(\alpha|s|+\imath \beta s) \varepsilon f}-\sum_{n=1}^{\infty} e^{-m_{1}|s| y-(\alpha|s|+\imath \beta s) \varepsilon f}\right) e^{-i\left(z-m_{2} y\right) s} d s \\
\tau_{x y}^{I I}=0 \\
\tau_{x z}^{I I}=0
\end{gathered}
$$




\section{Rough rigid-interface}

When the interfacing between the plate and half-space at $\mathrm{y}=\mathrm{Y}$ is rough-rigid, without loss of generality, on taking $T \longrightarrow 0$ i.e, $V=-1$ in equations (31)-(33) for med. I and in equations (34)-(36) for med. II. we will obtain the following results for displacements and stresses.

For med. I(i.e. , for $\leq y \leq Y)$

$$
\begin{gathered}
u^{\mathrm{I}}=\frac{P_{\mathrm{o}}}{2 \pi T_{1}} \int_{-\infty}^{\infty} \frac{\sin (s h)}{s|s|}\left(e^{-m_{1}|s| y}+\sum_{n=1}^{\infty}(-1)^{n} e^{-m_{1}|s|(2 n \varepsilon f-y)}-\right. \\
\left.\sum_{n=1}^{\infty}(-1)^{n} e^{-m_{1}|s|(2 n \varepsilon f+y)}\right) e^{-i\left(z-m_{2} y\right) s} d s \\
\tau_{x y}^{\mathrm{I}}=-\frac{P_{0}}{\pi}\left[\tan ^{-1} \frac{2 h m_{1} y}{\left(z-m_{2} y\right)^{2}+m_{1}^{2} y^{2}-h^{2}}-\sum_{n=1}^{\infty}(-1)^{n}\left(\tan ^{-1} \frac{2 h m_{1}(2 n \varepsilon f+y)}{\left(z-m_{2} y\right)^{2}+m_{1}^{2}(2 n \varepsilon f+y)^{2}-h^{2}}\right.\right. \\
\left.\left.+\tan ^{-1} \frac{2 h m_{1}(2 n \varepsilon f-y)}{\left(z-m_{2} y\right)^{2}+m_{1}^{2}(2 n \varepsilon f-y)^{2}-h^{2}}\right)\right] \\
\tau_{x z}^{\mathrm{I}}=-\frac{P_{0} m_{2}}{\pi}\left[\tan ^{-1} \frac{2 h m_{1} y}{\left(z-m_{2} y\right)^{2}+m_{1}^{2} y^{2}-h^{2}}-\sum_{n=1}^{\infty}(-1)^{n}\left(\tan ^{-1} \frac{2 h m_{1}(2 n \varepsilon f+y)}{\left(z-m_{2} y\right)^{2}+m_{1}^{2}(2 n \varepsilon f+y)^{2}-h^{2}}\right.\right. \\
\left.\left.+\tan ^{-1} \frac{2 h m_{1}(2 n \varepsilon f-y)}{\left(z-m_{2} y\right)^{2}+m_{1}^{2}(2 n \varepsilon f-y)^{2}-h^{2}}\right)\right]+\frac{m_{1} P_{0}}{2 \pi}\left[\log \frac{\left[\left(z-m_{2} y\right)+h\right]^{2}+\left(m_{1} y\right)^{2}}{\left[\left(z-m_{2} y\right)-h\right]^{2}+\left(m_{1} y\right)^{2}}\right. \\
\left.-\sum_{n=1}^{\infty}(-1)^{n}\left(\log \frac{\left[\left(z-m_{2} y\right)+h\right]^{2}+\left[m_{1}(2 n \varepsilon f+y)\right]^{2}}{\left[\left(z-m_{2} y\right)-h\right]^{2}+\left[m_{1}(2 n \varepsilon f+y)\right]^{2}}+\log \frac{\left[\left(z-m_{2} y\right)+h\right]^{2}+\left[m_{1}(2 n \varepsilon f-y)\right]^{2}}{\left[\left(z-m_{2} y\right)-h\right]^{2}+\left[m_{1}(2 n \varepsilon f-y)\right]^{2}}\right)\right]
\end{gathered}
$$

For med. II (i.e. $y>Y$ )

$$
\begin{aligned}
& u^{I I}=0 \\
& \tau_{x y}^{I I}=-\frac{2 P_{0}}{\pi}\left[\tan ^{-1} \frac{2 h\left(m_{1}^{\prime} y+\alpha \varepsilon f\right)}{\left(z-m_{2}^{\prime} y+\imath \beta \varepsilon f\right)^{2}+\left(m_{1}^{\prime} y+\alpha \varepsilon f^{2}\right)-h^{2}}\right. \\
&\left.+\sum_{n=1}^{\infty}(-1)^{n} \tan ^{-1} \frac{2 h\left(\left(2 n m_{1}+\alpha\right) \varepsilon f+m_{1}^{\prime} y\right)}{\left(z-m_{2}^{\prime} y+\imath \beta \varepsilon f^{2}\right)+\left(\left(2 n m_{1}+\alpha\right) \varepsilon f+m_{1}^{\prime} y\right)^{2}-h^{2}}\right] \\
& \tau_{x z}^{\mathrm{II}}=-\frac{2 P_{0} m_{2}^{\prime}}{\pi}\left(\tan ^{-1} \frac{2 h\left(m_{1}^{\prime} y+\alpha \varepsilon f\right)}{\left(z-m_{2}^{\prime} y+\imath \beta \varepsilon f\right)^{2}+\left(m_{1}^{\prime} y+\alpha \varepsilon f\right)^{2}-h^{2}}\right. \\
&+\left.\sum_{n=1}^{\infty}(-1)^{n} \tan ^{-1} \frac{2 h\left(\left(2 n m_{1}+\alpha\right) \varepsilon f+m_{1}^{\prime} y\right)}{\left(z-m_{2}^{\prime} y+i \beta \varepsilon f^{2}+\left(\left(2 n m_{1}+\alpha\right) \varepsilon f+m_{1}^{\prime} y\right)^{2}-h^{2}\right.}\right) \\
&+\frac{P_{0} m_{1}^{\prime}}{\pi}\left(\log \frac{\left(z-m_{2}^{\prime} y+i \beta \varepsilon f+h\right)^{2}+\left(m_{1}^{\prime} y+\alpha \varepsilon f\right)^{2}}{\left(z-m_{2}^{\prime} y+i \beta \varepsilon f-h\right)^{2}+\left(m_{1}^{\prime} y+\alpha \varepsilon f\right)^{2}}+\right. \\
&\left.+\sum_{n=1}^{\infty}(-1)^{n} \log \frac{\left(z-m_{2}^{\prime} y+i \beta \varepsilon f+h\right)^{2}+\left(m_{1}^{\prime} y+\left(2 n m_{1}+\alpha\right) \varepsilon f\right)^{2}}{\left(z-m_{2}^{\prime} y+i \beta \varepsilon f-h\right)^{2}+\left(m_{1}^{\prime} y+\left(2 n m_{1}+\alpha\right) \varepsilon f\right)^{2}}\right)
\end{aligned}
$$




\subsection{Particular cases}

\section{Orthotropic elastic layered half-space:}

By putting $c_{56}=0$ i.e $m_{1}=m_{3}=\sqrt{\frac{c_{55}}{c_{66}}}$ and $m_{2}=0$ in the equations (31)-(36), we will get the deformation of an orthotropic elastic layered half space due to strip loading.

Isotropic elastic layered half space:

By putting $c_{56}=0$ and $c_{66}=c_{55}=\mu$ i.e. $m_{2}=0$ and $m_{1}=m_{3}=1$ in the equations (31)-(36), we will get the deformation of an isotropic elastic layered half space due to strip loading.

\subsection{Special cases}

(i) By putting $c_{56}=0$ i.e $m_{2}=0, m_{1}=m_{3}=\sqrt{\frac{c_{55}}{c_{66}}}=m$, and $c_{56}^{\prime}=0, c_{66}^{\prime}=c_{55}^{\prime}=\mu$, i.e $m_{2}^{\prime}=0, m_{1}^{\prime}=m_{3}^{\prime}=1$ in equations (31)-(36) we will obtain the system of equations

$$
\begin{gathered}
u^{\mathrm{I}}=\frac{P_{0}}{\pi T_{1}} \int_{-\infty}^{\infty} \frac{\sin (s h)}{s|s|}\left(e^{-m|s| y}+\sum_{n=1}^{\infty} V^{n} e^{-m|s|(2 n \varepsilon f-y)}-\sum_{n=1}^{\infty} V^{n} e^{-m|s|(2 n \in f+y)}\right) e^{-i z s} d s \\
\tau_{x y}^{\mathrm{I}}=-\frac{P_{0}}{\pi}\left[\tan ^{-1} \frac{2 h m y}{z^{2}+m^{2} y^{2}-h^{2}}-\sum_{n=1}^{\infty} V^{n}\left(\tan ^{-1} \frac{2 h m(2 n \varepsilon f+y)}{z^{2}+m^{2}(2 n \varepsilon f+y)^{2}-h^{2}}+\tan ^{-1} \frac{2 h m(2 n \varepsilon f-y)}{z^{2}+m^{2}(2 n \varepsilon f-y)^{2}-h^{2}}\right)\right] \\
\tau_{x z}^{\mathrm{I}}=\frac{m_{1} P_{0}}{2 \pi}\left[\log \frac{[z+h]^{2}+(m y)^{2}}{[z-h]^{2}+(m y)^{2}}-\sum_{n=1}^{\infty} V^{n}\left(\log \frac{[z+h]^{2}+[m(2 n \varepsilon f+y)]^{2}}{[z-h]^{2}+[m(2 n \varepsilon f+y)]^{2}}+\log \frac{[z+h]^{2}+[m(2 n \varepsilon f-y)]^{2}}{[z-h]^{2}+[m(2 n \varepsilon f-y)]^{2}}\right)\right] \\
u^{\mathrm{II}}=\frac{2 P_{0}}{\pi T_{1}}\left(\frac{T}{T+1}\right) \int_{-\infty}^{\infty} \frac{\sin (s h)}{s|s|}\left(e^{-m|s| y-((m-1)|s|) \varepsilon f}+\sum_{n=1}^{\infty} V^{n} e^{-m \mid s((2 n \varepsilon f+y)-((m-1)|s|) \varepsilon f)}\right) e^{-i(z) s} d s \\
\tau_{x y}^{\mathrm{II}}=-\frac{2 P_{0}}{\pi(1+T)}\left[\tan ^{-1} \frac{2 h(y+(m-1) \varepsilon f)}{(z)^{2}+(y+(m-1) \varepsilon f)^{2}-h^{2}}+\sum_{n=1}^{\infty} V^{n} \tan ^{-1} \frac{2 h((2 n m+(m-1)) \varepsilon f+y)}{(z)^{2}+((2 n m+(m-1)) \varepsilon f+y)^{2}-h^{2}}\right] \\
\tau_{x z}^{\mathrm{II}}=\frac{P_{0}}{\pi(1+T)}\left(\log \frac{(z+h)^{2}+(y+(m-1) \varepsilon f)^{2}}{(z-h)^{2}+(y+(m-1) \varepsilon f)^{2}}+\sum_{n=1}^{\infty} V^{n} \log \frac{(z+h)^{2}+(y+(2 n m+(m-1)) \varepsilon f)^{2}}{(z-h)^{2}+(y+(2 n m+(m-1)) \varepsilon f)^{2}}\right)
\end{gathered}
$$

which is similar to Madan ${ }^{(10)}$.

(ii) By taking the lower monoclinic elastic half space as a simple base the obtained results (31)-(36), (37)-(41) and (42)-(47) can similar to $\operatorname{Madan}^{(7)}$ for all three different boundary conditions "perfectly welded interfacing, smooth-rigid interfacing, and rough-rigid interfacing" respectively.

\section{Numerical results}

Here, we want to describe the effect of rectangular irregularity on the stresses due to strip load $P_{0}=10^{7}$ dynes $/ \mathrm{cm}^{-2}$ acting on $((z \mid \leq h)$ the upper layer of monoclinic elastic plate connected with monoclinic elastic half space. For numerical calculation, we take the following data for elastic constants from Tiersten ${ }^{(13)}$.

(a) For monoclinic elastic layer,

$$
\begin{gathered}
c_{55}^{\prime}=94 \times 10^{9} \mathrm{~N} / \mathrm{m}^{2}, \\
c_{66}^{\prime}=93 \times 10^{9} \mathrm{~N} / \mathrm{m}^{2}, \\
c_{56}^{\prime}=-11 \times 10^{9} \mathrm{~N} / \mathrm{m}^{2},
\end{gathered}
$$

(b) For monoclinic elastic half-space,

$$
\begin{aligned}
& c_{55}^{\prime \prime}=57.94 \times 10^{9} \mathrm{~N} / \mathrm{m}^{2}, \\
& c_{66}^{\prime \prime}=39.88 \times 10^{9} \mathrm{~N} / \mathrm{m}^{2}, \\
& c_{56}^{\prime \prime}=-17.91 \times 10^{9} \mathrm{~N} / \mathrm{m}^{2}
\end{aligned}
$$


Figures 2, 3, 4 and 5 represent the variation of shearing stresses $\tau_{x y}$ and $\tau_{x z}$ for Med. I and Figures 6, 7 and 8 represent the variation of shearing stresses $\tau_{x y}$ and $\tau_{x z}$ for Med. II in perfectly welded contact, with horizontal distance ' $z$ ' and for strip size ' $h$ ' at depth level $y$. All figures (2)-(8) in perfectly welded contact are calculated for $T_{1}=T_{1}^{\prime}$. Figures 9,10 and 11 represent the variation of shearing stresses $\tau_{x y}$ and $\tau_{x z}$ in smooth-rigid contact for Med. I, for different values of $h=1,1.5,2$ at $y=1$ respectively. Figures 12 and 13 represent the variation of shearing stress $\tau_{x y}$ and $\tau_{x z}$ in rough-rigid contact for Med. I and Figures 14, 15, 16 and 17 in case of Med. II. In figures (9)-(17) both shearing stresses $\tau_{x y}$ and $\tau_{x z}$ have been plotted for all series terms by taking the sum up to first ten terms of the series. As per our consideration, it is mentioned that the irregularity is present in the lower half space. It has been observed graphically that the stresses for Med. I (i.e monoclinic elastic layer) are affected by the change of loaded strip size but not by irregularity and this observation prevails in all the three interfacing boundary conditions. So, it has been concluded that the change in the size of irregularity has affected the stresses in lower half space. Also It has been found that the stresses $\tau_{x y}$ and $\tau_{x z}$ for Med.I at $(\mathrm{h}=-0.25,-0.5,0.25,0.5)$ have no discontinuity and the discontinuity comes in graphs for $\mathrm{h}>0.5$, shown in figures (2)-(5) and the same has been observed for stresses in smooth or rough-rigid contacts. Moreover, it has been observed that the stresses for Med. II (i.e lower half space) have discontinuities due to irregularity as well as strip size $((z \mid \leq h)$ for $\mathrm{h} \geq 1$. Same pattern has been observed in lower half space, if $a=0$ in absence of rectangular irregularity (i.e in case of regular half space) with $(h \mid \leq 0.5$ the stresses are continuous, clearly in figures (8), (16). Graphically, it has been concluded that in both mediums the variation in shearing stresses is affected by anisotropy of material, size of the strip and size of an irregularity. Also, the difference between the shearing stresses in magnitude decreases as horizontal distance increases.

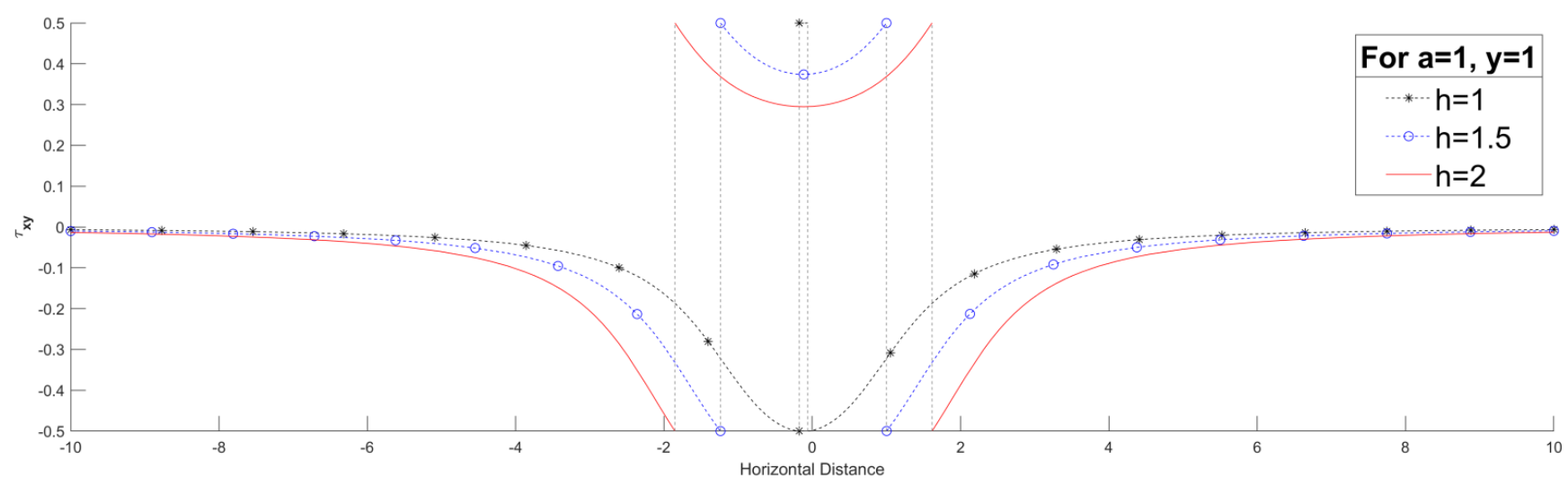

Fig 2. Variation of the stress component $\tau_{x y}$ in perfectly welded contact with horizontal distance $z$, for Med. I

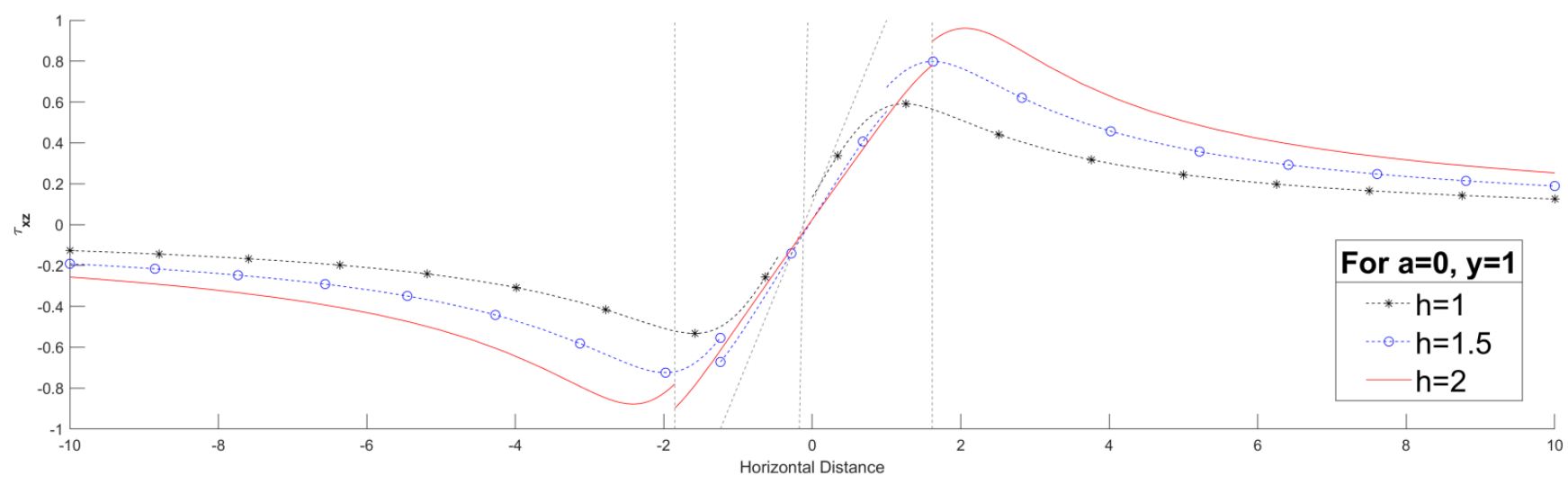

Fig 3. Variation of the stress component $\tau_{x z}$ in perfectly welded contact with horizontal distance $z$, for Med. I 


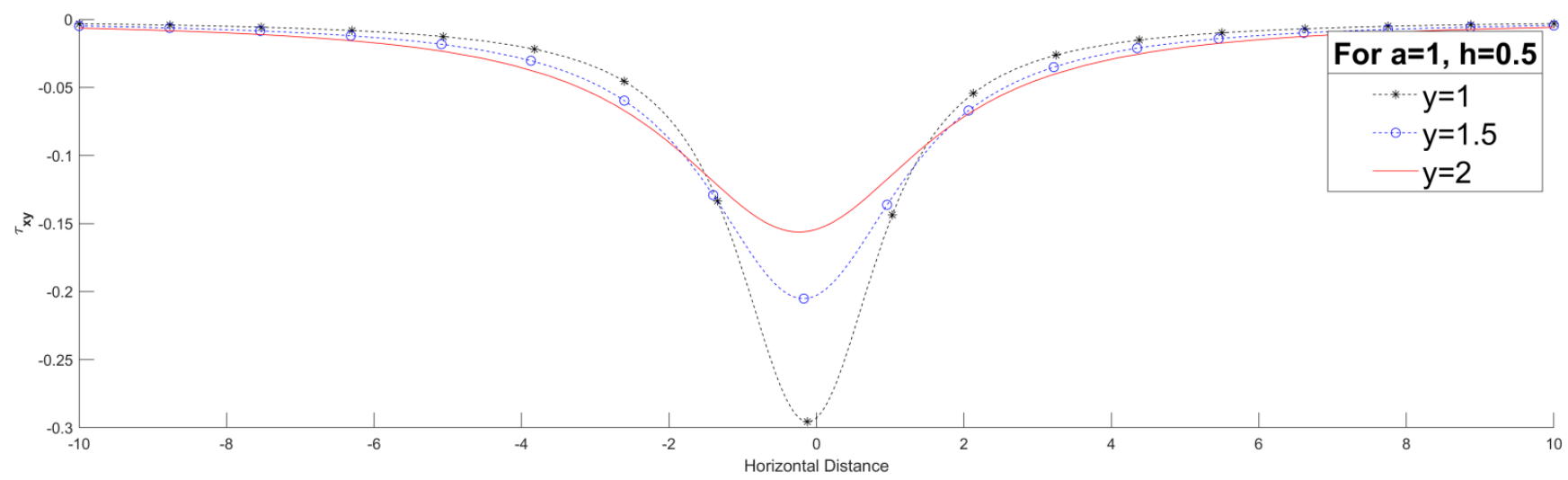

Fig 4. Variation of the stress component $\tau_{x y}$ in perfectly welded contact with horizontal distance $z$, for Med. I

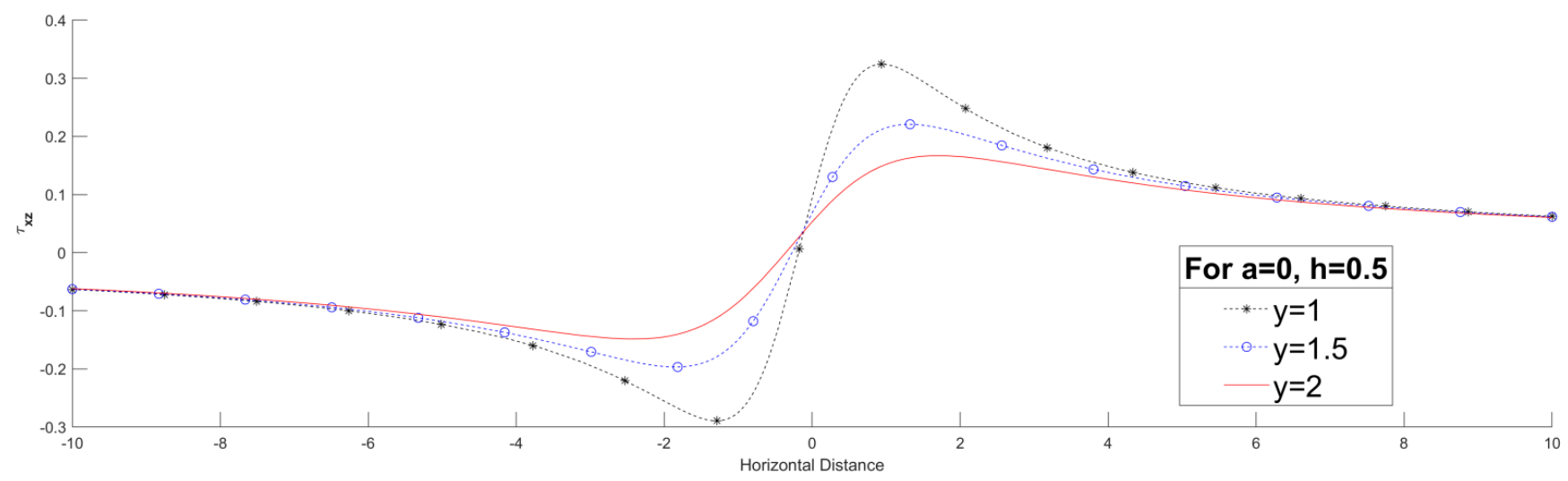

Fig 5. Variation of the stress component $\tau_{x z}$ in perfectly welded contact with horizontal distance $z$, for Med. I

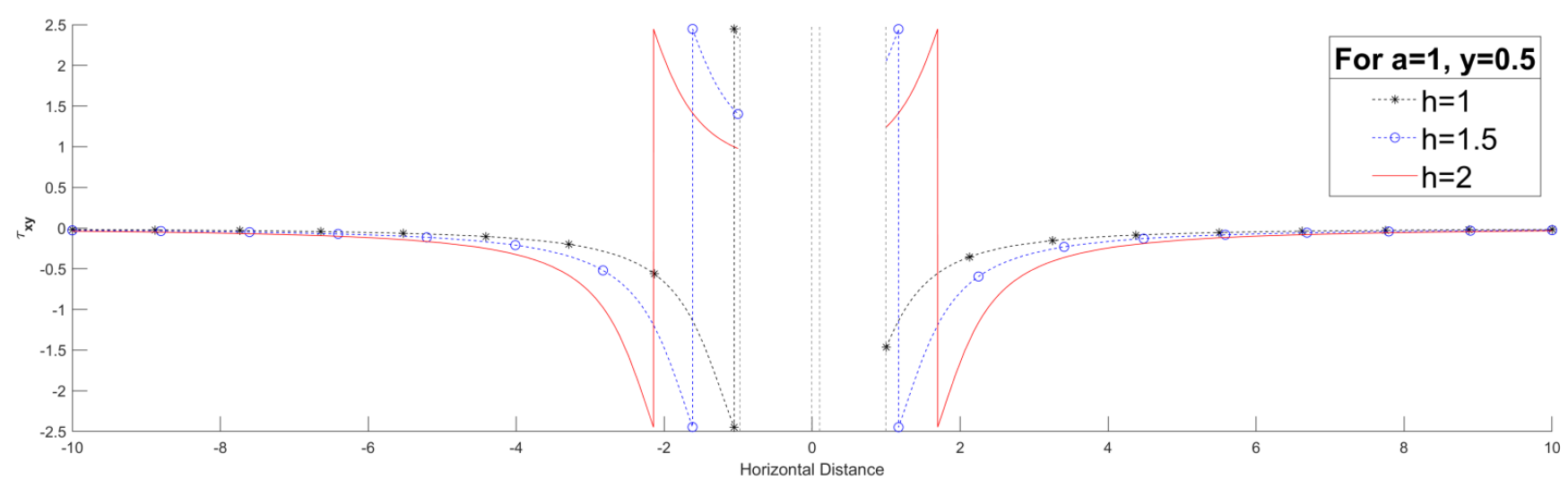

Fig 6. Variation of the stress component $\tau_{x y}$ in perfectly welded contact with horizontal distance $z$, for Med. II 


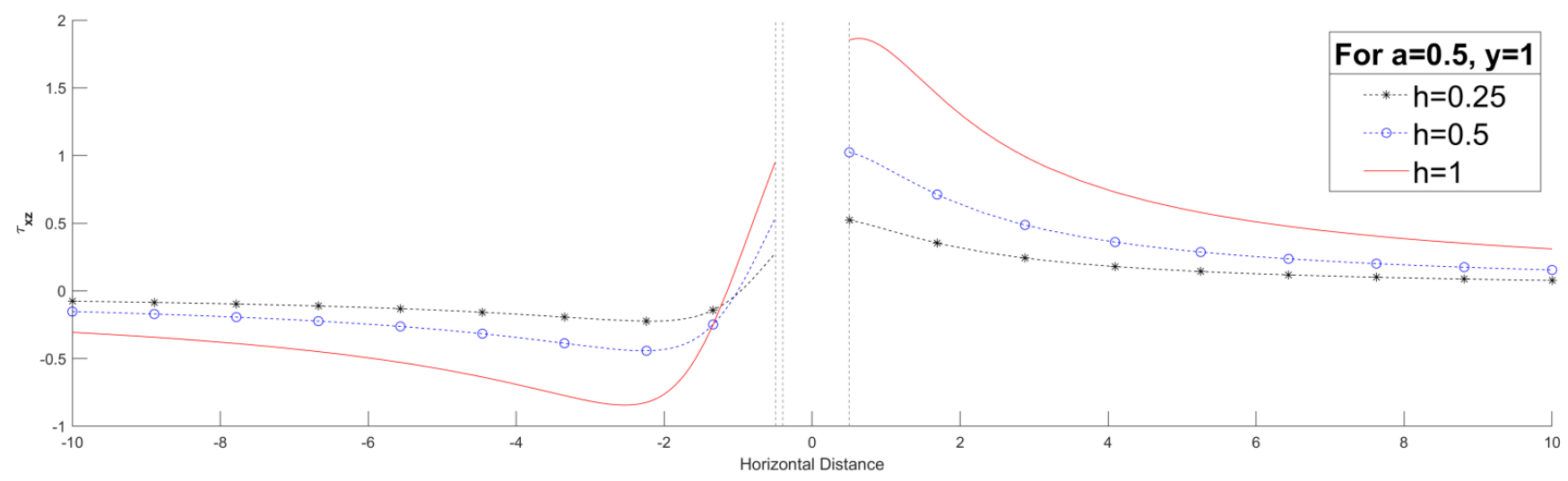

Fig 7. Variation of the stress component $\tau_{x z}$ in perfectly welded contact with horizontal distance $z$, for Med. II

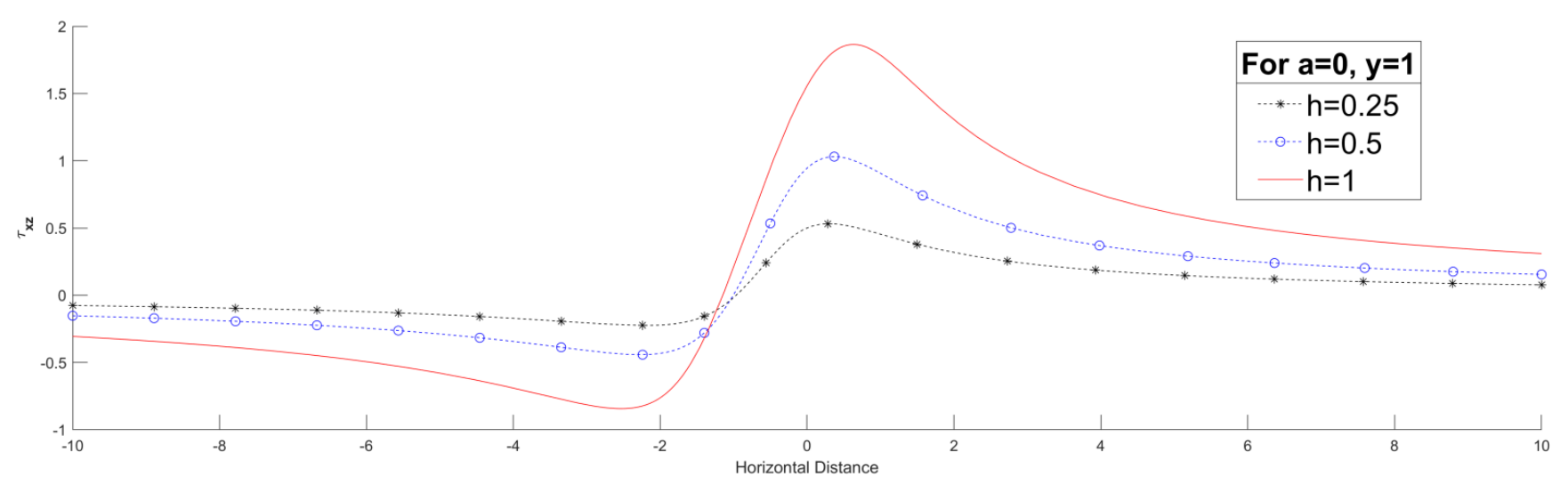

Fig 8. Variation of the stress component $\tau_{x z}$ in perfectly welded contact with horizontal distance $z$, for Med. II

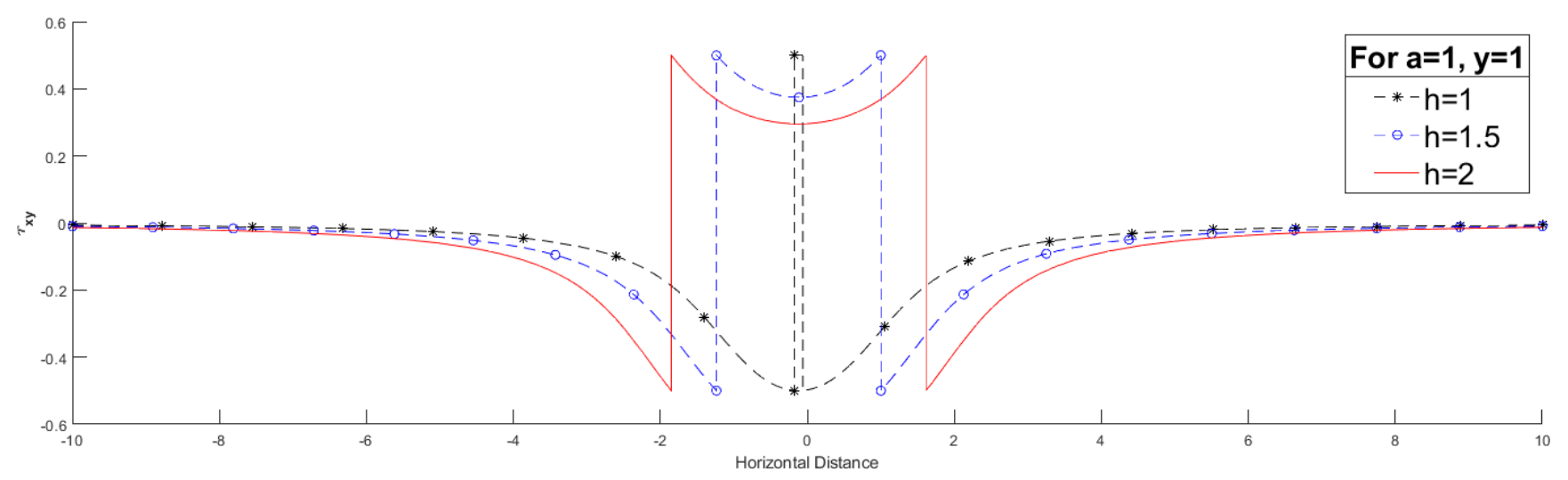

Fig 9. Variation of the stress component $\tau_{x y}$ in smooth-rigid contact with horizontal distance $z$, for Med. I 


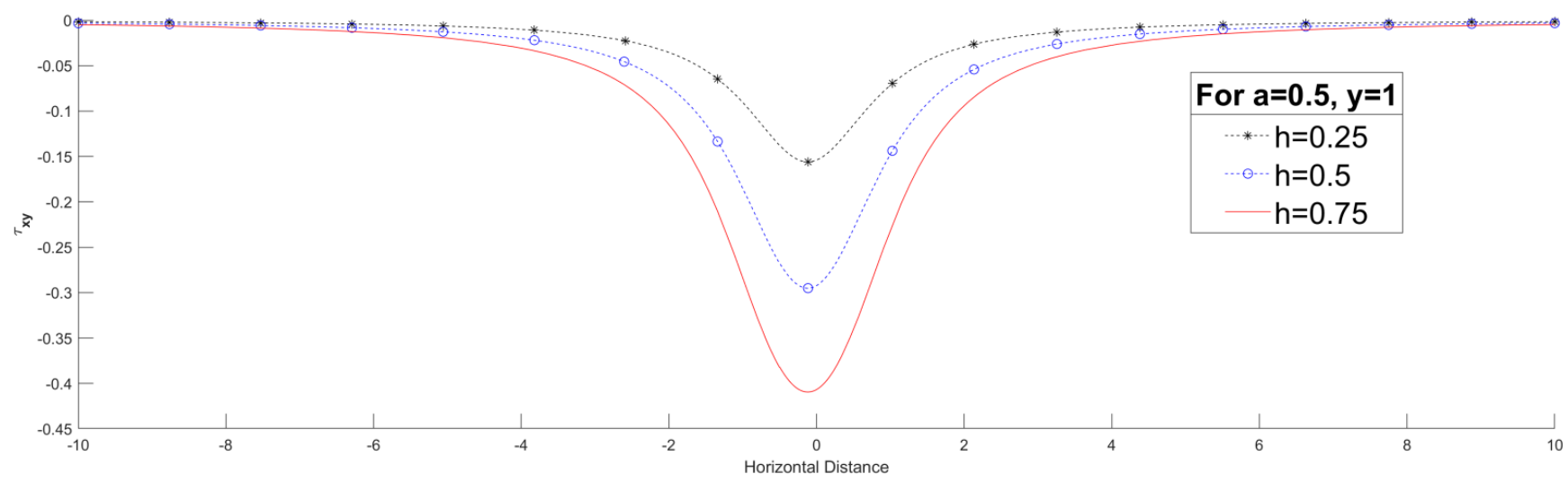

Fig 10. Variation of the stress component $\tau_{x y}$ in smooth-rigid contact with horizontal distance $z$, for Med. I

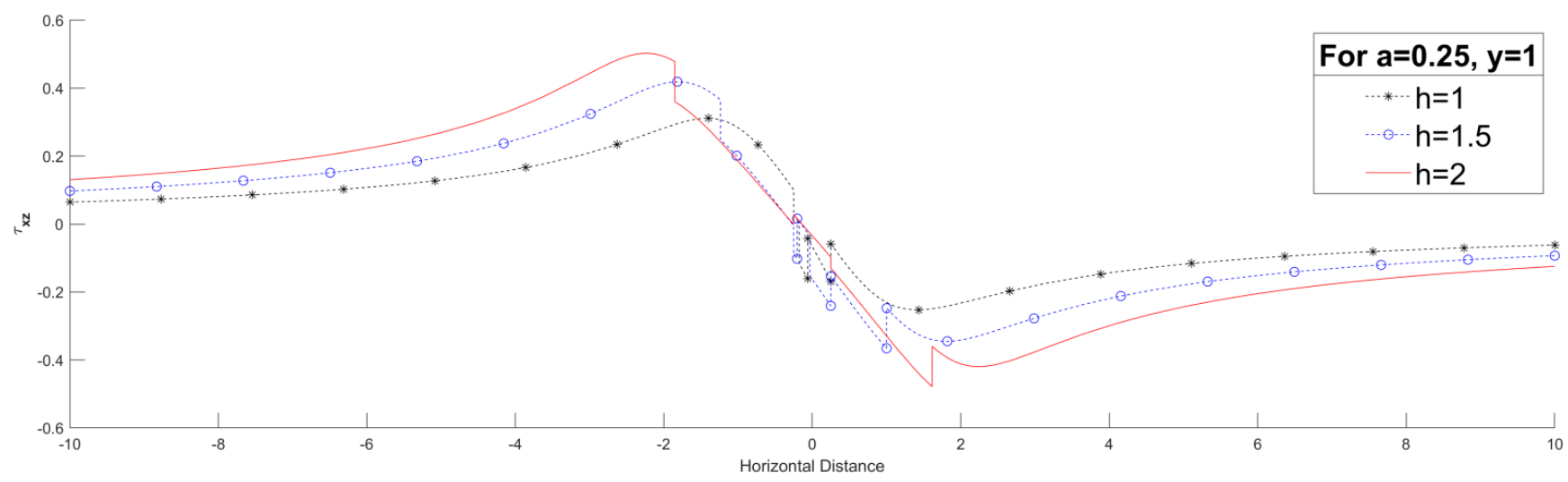

Fig 11. Variation of the stress component $\tau_{x z}$ in smooth-rigid contact with horizontal distance $z$, for Med. I

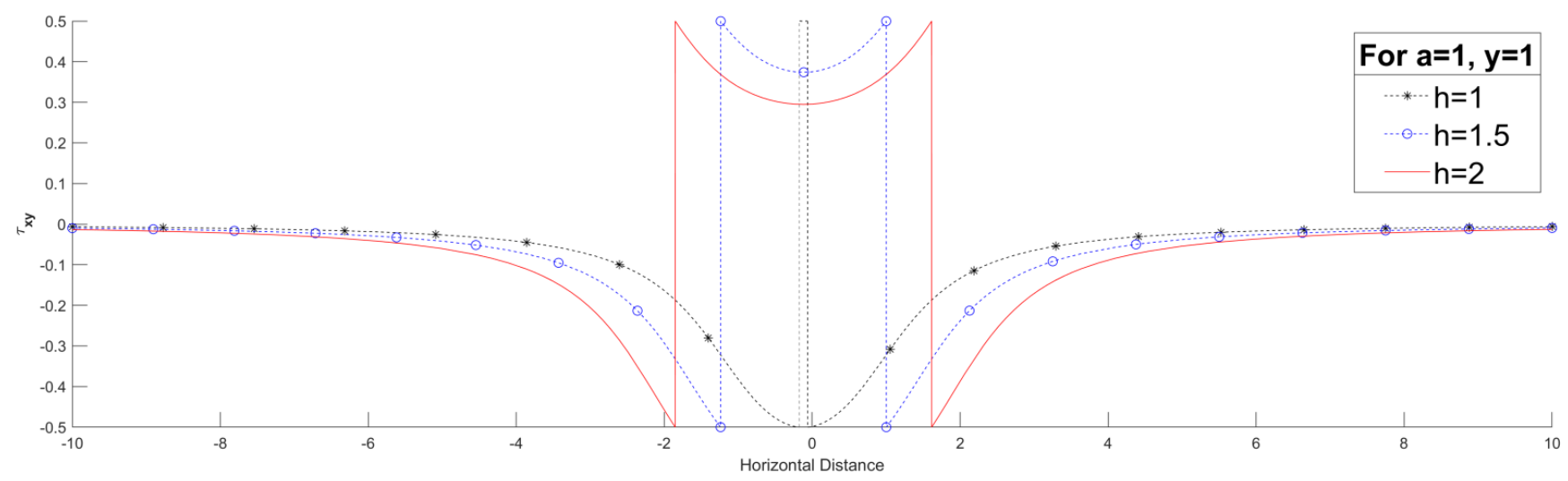

Fig 12. Variation of the stress component $\tau_{x y}$ in rough-rigid contact with horizontal distance $z$, for Med. I 


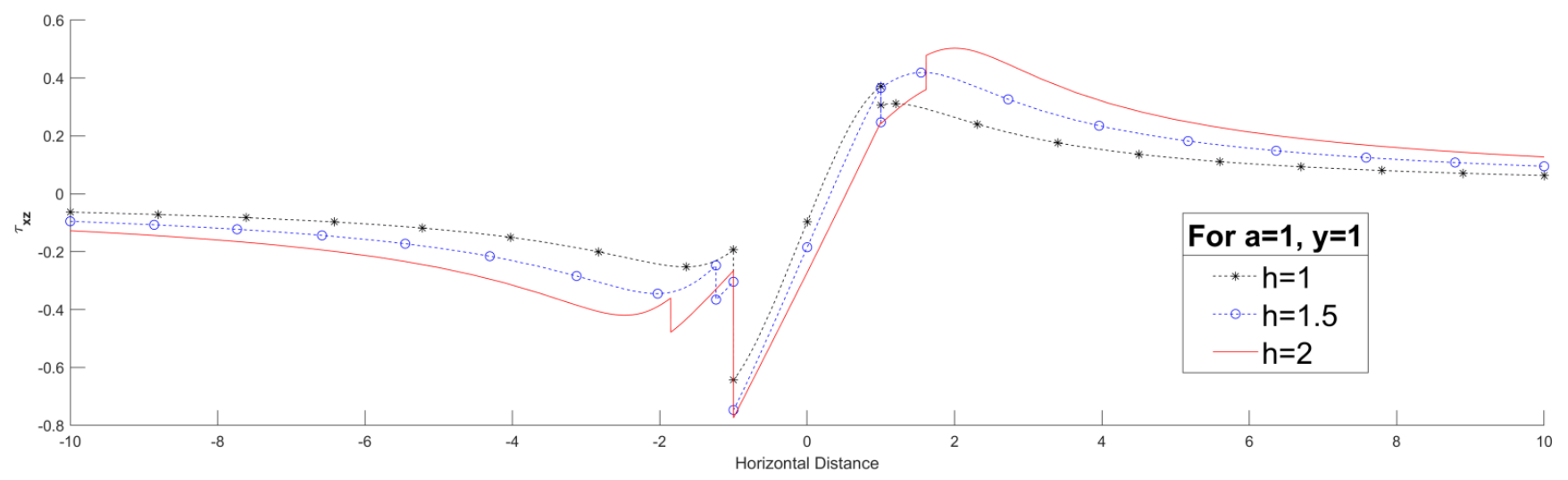

Fig 13. Variation of the stress component $\tau_{x z}$ in rough-rigid contact with horizontal distance $z$, for Med.I

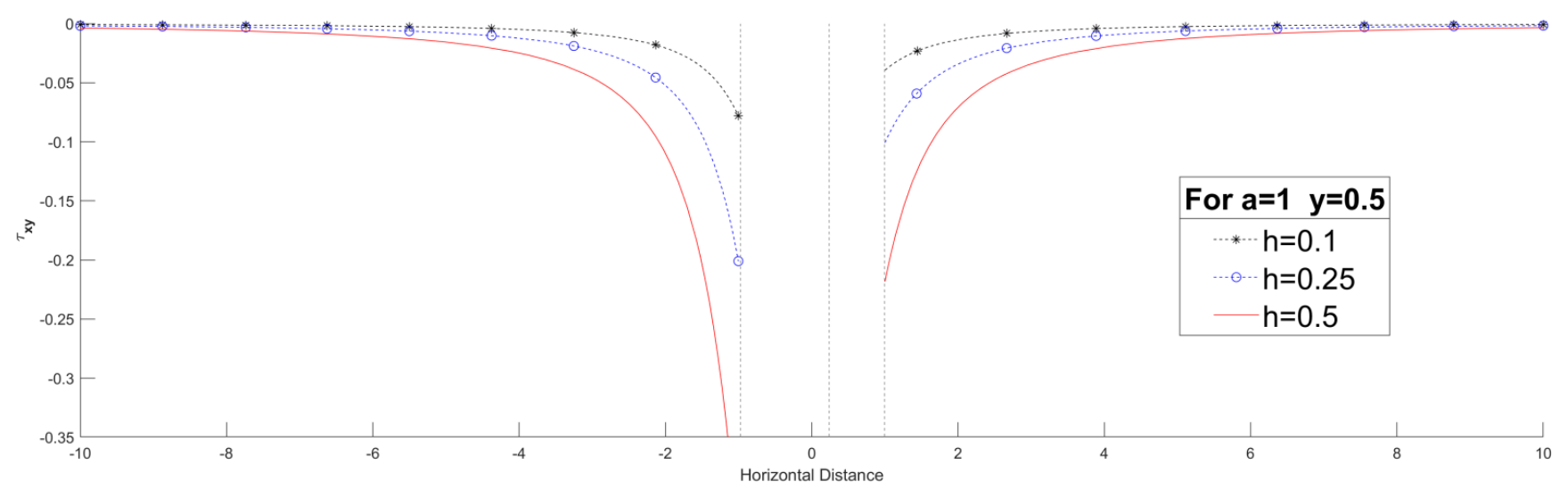

Fig 14. Variation of the stress component $\tau_{x y}$ in rough-rigid contact with horizontal distance $z$, for Med. II

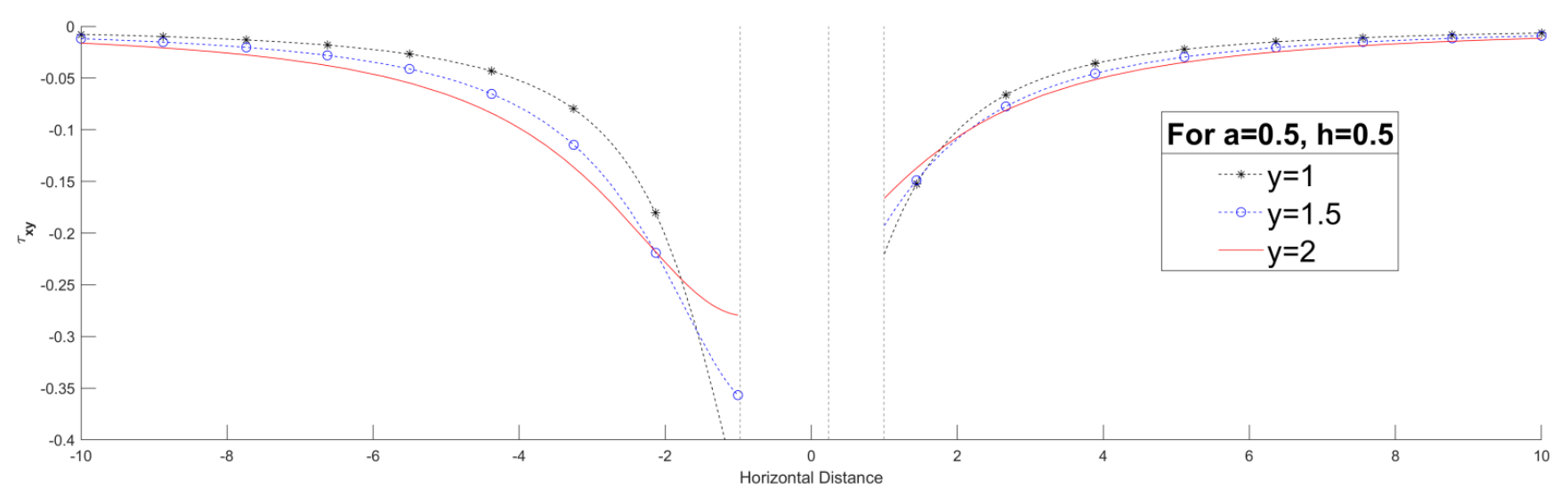

Fig 15. Variation of the stress component $\tau_{x y}$ in rough-rigid contact with horizontal distance $z$, for Med. II 


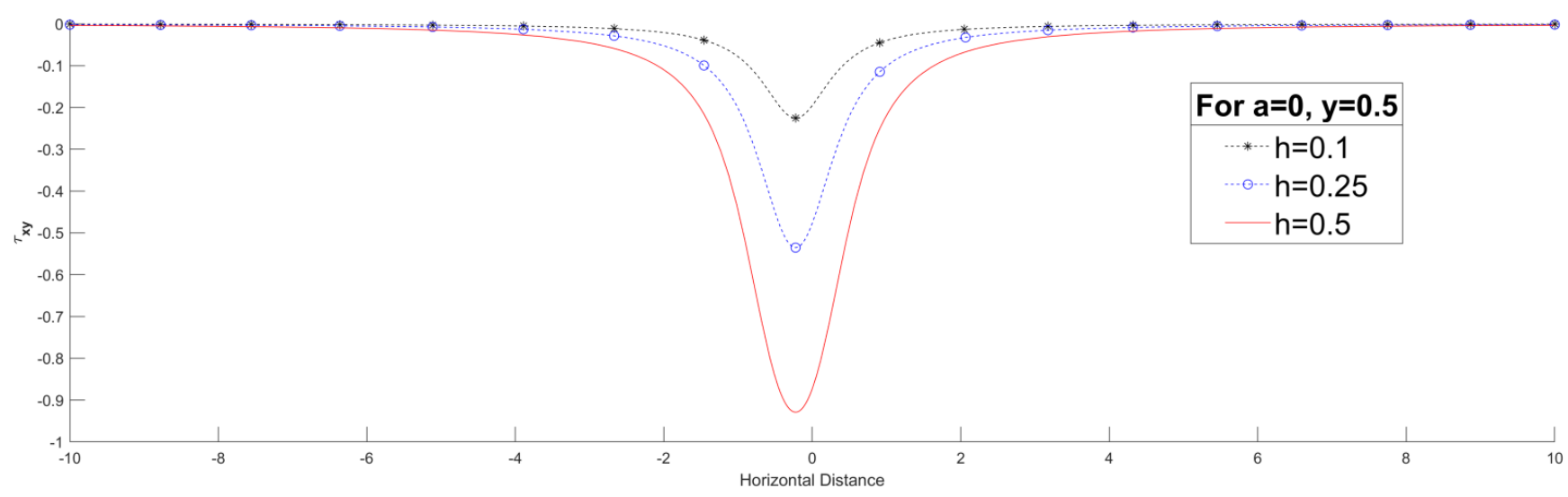

Fig 16. Variation of the stress component $\tau_{x y}$ in rough-rigid contact with horizontal distance $z$, for Med. II

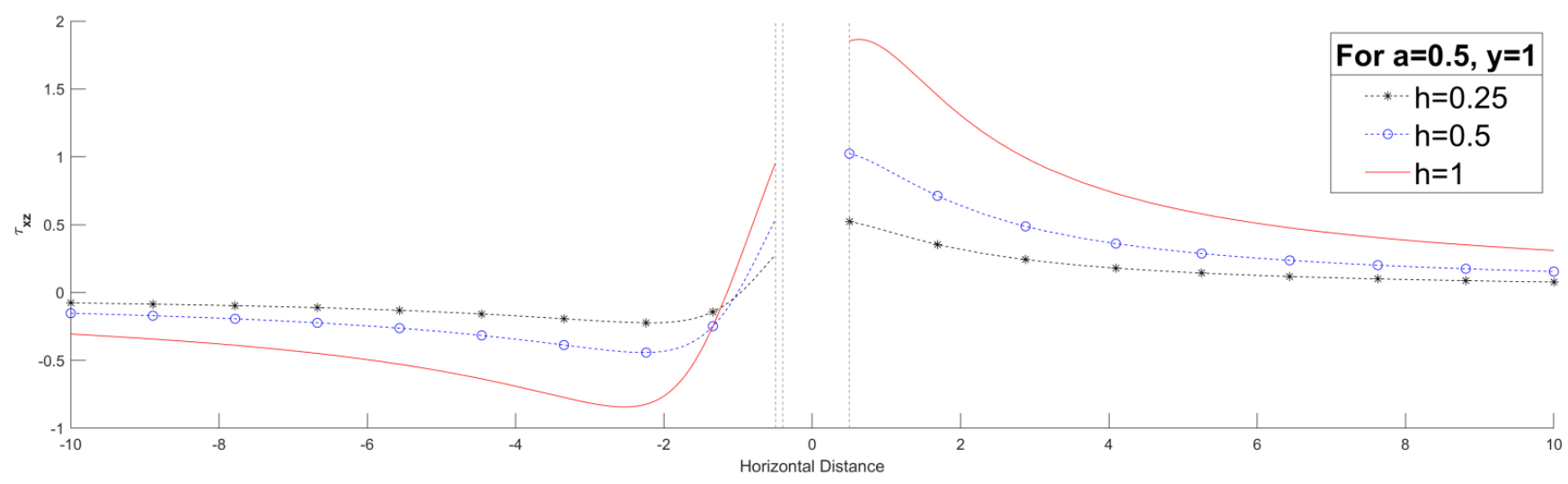

Fig 17. Variation of the stress component $\tau_{x z}$ in rough-rigid contact with horizontal distance $z$, for Med. II

\section{Conclusions}

The closed form expressions for the stresses in an elastic model consisting of monoclinic elastic layer lying over an irregular monoclinic elastic half space due to shear strip load has been obtained. Graphically, it has been concluded that the stresses in an infinite monoclinic layer interface with an irregular monoclinic half space are significantly affected by the presence of an irregularity and also by anisotropy of the elastic medium as a result of shear load. The irregularity may be rectangular, triangular, parabolic etc. Also the difference between the shearing stresses in magnitude decreases as horizontal distance increases. The results obtained are useful to study the static deformation near the surface of the earth where lithostatic pressure have disjoint cracks perpendicular to the maximum compressional stress. The corresponding results obtained by Madan ${ }^{(7)}$ and by Madan ${ }^{(10)}$ can be obtained from our results as particular cases.

\section{Acknowledgement}

One of the authors (Savita) is thankful to HRDG Council of Scientific \& Industrial Research for sanctioning SRF scholarship with File No. (09/1063/0012/2017). The authors are also great thankful to the unknown reviewers.

\section{References}

1) Crampin S. Suggestions for a consistent terminology for seismic anisotropy. Geophysical Prospecting. 1989;37(7):753-770. Available from: https: //dx.doi.org/10.1111/j.1365-2478.1989.tb02232.x.

2) Pan E. Static response of a transversely isotropic and layered half-space to general dislocation sources. Physics of the Earth and Planetary Interiors. 1989;58(2-3):103-117. Available from: https://dx.doi.org/10.1016/0031-9201(89)90046-0.

3) Garg NR, Singh SJ. Quasi-static response of a layered half space to surface loads. Indian Journal of pure and applied Mathematics. 1989;20:621-631. Available from: http://www.new.dli.ernet.in/rawdataupload/-upload. 
4) Madan DK, Garg NR. Static deformation of an orthotropic horizontal elastic layer coupling in different way to a base due to a very long inclined strike slip fault embedded in the layer. Indian Journal of pure and applied Mathematics. 1997;28:697-712.

5) Garg NR, Madan DK, Sharma RK. Two-dimensional deformation of an orthotropic elastic medium due to seismic sources. Physics of the Earth and Planetary Interiors. 1996;94(1-2):43-62. Available from: https://dx.doi.org/10.1016/0031-9201(95)03095-6.

6) Singh K, Madan DK, Goel A, Garg NR. Two-dimensional static deformation of an anisotropic medium. Sadhana. 2005;30:565-583. Available from: https://dx.doi.org/10.1007/bf02703280.

7) Madan DK, Chugh KS, Singh. Stresses in an anisotropic Elastic Plate due to Strip-Loading. International Journal of Mechanics. 2011;5:57-62. Available from: https://www.researchgate.net-/publication/275408846.

8) Madan ADK, Dahiya S, Chugh. Static Response of Transversely Isotropic Elastic Medium with irregularity in the medium. International Journal of Mechanical Engineering. 2012;2:1-11.

9) Madan DK, Gabba A. 2-Dimensional Deformation of an irregular Orthotropic Elastic Medium. IOSR Journal of mathematics. 2016;12:101-113. Available from: https://doi.org/10.9790/5728120405101113.

10) Madan PDK, Arya NR, Garg K, Singh. Stresses in an orthotropic elastic layer lying over in irregular isotropic elastic half-space. International Journal of Current Research and Review. 2017;09(04):15-20.

11) Chugh S. Contour Map Study of Stresses in a Transversely Isotropic Elastic Plate due to Strip-Loading. International Research Journal of Engineering and Technology. 2020;07(06):3173-3179. Available from: https://www.irjet.net/archives/V7/i6/IRJET-V7I6592.

12) Verma RC, Rani S, Singh SJ. Deformation of a poroelastic layer overlying an elastic half-space due to dip-slip faulting. International Journal for numerical and analytical methods in Geomechanics. 2016;40:391-405. Available from: https://doi.org/10.1002/nag.2410.

13) Tiersten HF. Linear Piezoelactric plate vibrations. New York. Plenum Pres.. 1969. 